\title{
Performance analysis of impurity added benzene based single-electron transistor
}

\author{
Anurag Srivastava • B. Santhibhushan • \\ Pankaj Dobwal
}

Received: 26 August 2012 / Accepted: 7 January 2013/Published online: 6 February 2013

(c) The Author(s) 2013. This article is published with open access at Springerlink.com

\begin{abstract}
We have analyzed the impurity added benzene based single-electron transistor (SET) using the ab initio approach, based on density functional theory and non-equilibrium Green's function. Boron and nitrogen has been added as impurities either by replacing the last carbon atom or last hydrogen in the benzene. The system has been modeled in such a way that the impurity added benzene is placed above the gate dielectric in coulomb blockade regime between source and drain electrodes for weak coupling. The charging energies of the system have been calculated and discussed in both the isolated as well as SET environments. The conductance dependence of SET on bias potential and gate voltage has been verified through charge stability diagrams.
\end{abstract}

Keywords: Impurity added benzene (IAB) -

Single-electron transistor (SET) - Charge states ·

Charging energy $\cdot$ Charge stability diagram

\section{Introduction}

A conventional transistor is a three terminal solid-state semiconductor device which can be used for several applications in electronics. The basic use of a transistor is as a switch but with the technology shrinking, the researchers have started working to produce the smaller and faster switch. As stated by Moore's law, for every 2 years the number of transistors on a chip should be doubled but as the transistor size is entering into the

A. Srivastava $(\bowtie) \cdot$ B. Santhibhushan $\cdot$ P. Dobwal Advanced Materials Research Group, Computational Nanoscience and Technology Lab, ABV-Indian Institute of Information Technology and Management, Gwalior, MP, India e-mail: profanurag@gmail.com nanometer regime, transistors are suffering from several second-order effects and dominance of leakage current (Mollick 2006). To meet out these challenges, the singleelectron transistors (SET) are likely to become an alternative for producing smaller and faster switches. The ultra small size of SET will allow the designers to integrate more number of transistors on a chip. In recent years, researchers have started focusing on the use of the organic molecules as island in the single-electron transistor (Stokbro 2010; Brandbyge et al. 2002; Neaton et al. 2006; Seldenthuis et al. 2010; Parashar et al. 2012).

\section{Single-electron transistor (SET): Theory}

Single-electron transistor is a device based on the concept of one-by-one electron tunneling through island utilizing the coulomb blockade effect. It consists of two electrodes named as drain and source, connected through tunnel junctions to a common electrode with a low self-capacitance, known as the island. The electrical potential of the island can be tuned by a third electrode, known as the gate which is capacitively coupled to the island as shown in Fig. 1. The only way to an electron to travel from one electrode to another is tunneling through the island. (Kumar and Kaur 2010; Fulton and Dolan 1987; Stokbro 2010; Grabert and Devoret 1992).

For an electron to tunnel onto the island, its energy must be equal to the Coulomb energy $\mathrm{e}^{2} / 2 \mathrm{C}$, where $C$ is the selfcapacitance of the island defined as

$C=\frac{e^{2}}{\Delta E}$

Here, $\Delta E$ is the separation between the energy levels of the island. 


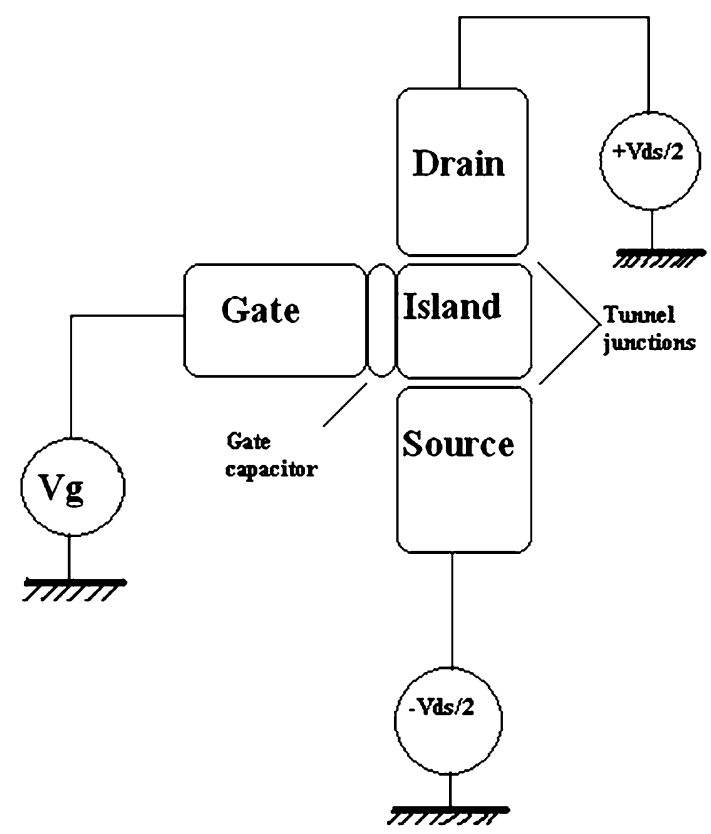

Fig. 1 Schematic of single electron transistor

Capacitance of the island must be $<10^{-17}$ Farads and, therefore, its size should not be $>10 \mathrm{~nm}$. In SET at zero gate and bias voltages, the electrons do not have enough energy to enter the island and hence, there will be no current flow. When the bias voltage between the source and drain increases, electron can pass through the island as its energy reaches the Coulomb energy. This effect is known as the Coulomb blockade. The critical voltage needed to transfer an electron onto the island, equal to $e / C$, is called as Coulomb gap voltage. To achieve the Coulomb blockade, three criteria have to be met:

1. The bias voltage must be lower than the elementary charge divided by the self-capacitance of the island: $V_{\text {bias }}<\frac{e}{C}$.

2. The thermal energy in the source contact plus the thermal energy in the island i.e., $K_{B} T$, must be below the charging energy: $K_{B} T<\frac{e^{2}}{C}$, or else the electron will be able to pass the island via thermal excitation.

3. And the tunneling resistance, $R_{\mathrm{t}}$, should be greater than $\frac{h}{e^{2}}$ which is derived from Heisenberg's uncertainty principle.

The Coulomb blockade tries to alleviate any leak by current during the off state of the single-electron transistor (Fulton and Dolan 1987; Grabert and Devoret 1992; Altshuler et al. 1991).

In SET environment, electron flow happens because of the difference of agenda of Fermi functions in the two contacts where one keeps filling up the level(s) while the other empties them, the result of this process is a net flow

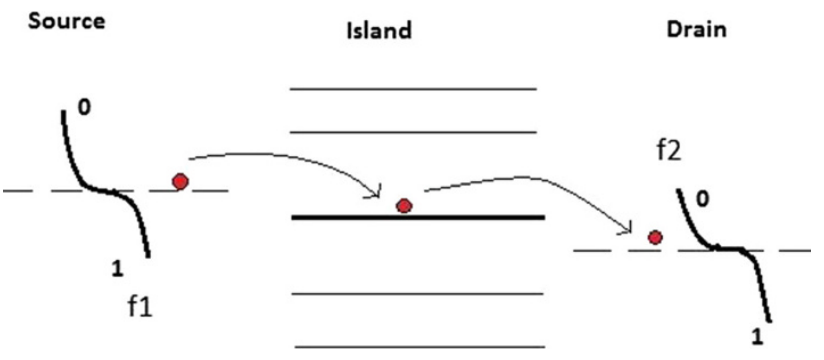

Fig. 2 Electron tunneling through the levels

of current. Here, all the levels do not conduct, only some levels support the flow of electrons. From Fig. 2, one can see that the two Fermi functions that levels deep down and way above do not contribute to current, because at those levels $\mathrm{f} 1$ and $\mathrm{f} 2$ have the same values of 1 and 0 's. The maximum conductance of SET is also called as the Quantum of conductance, $G_{\max }=2 q^{2} / h$. Correspondingly, the minimum resistance value is $R_{\min }=h / 2 q^{2}$.

The current in the single-electron transistor and the average number of electrons can be given by the expressions:

$I=\frac{q}{h} \frac{\gamma_{1} \gamma_{2}}{\gamma_{1+} \gamma_{2}}\left(f_{1}-f_{2}\right)$

$N=\frac{\gamma_{1} f_{1}+\gamma_{2} f_{2}}{\gamma_{1}+\gamma_{2}}$

Here $\gamma_{1}$ and $\gamma_{2}$ are the parameters which explains the electron escaping from source or island. On dividing these parameters by $h$, the rate of escape can be explained. The last factor in the current expression tells that current flows if there is a difference between the Fermi functions of contacts. If both $\mathrm{f} 1$ and $\mathrm{f} 2$ are equal, then current will be zero (Datta 2004).

The validity of above expressions lies with the fact that applied voltage must be small. If the coupling is stronger, then we have to take into account the concept of 'Broadening'. If a strong voltage is applied to the contacts, then the sharp discrete level broadens out and becomes a continuum of states as showed in Fig. 3, the sum of which is still one level and can accommodate one electron. In Fig. 3, if the energy range $d E$ has density of states $D(E)$, then the expressions for current and the average number of electrons given in the Eq.(1) and Eq.(2) can be expressed as, (Datta 2004).

$I=\frac{-q}{h} \int D(E) d E \frac{\gamma_{1} \gamma_{2}}{\gamma_{1}+\gamma_{2}}\left(f_{1}-f_{2}\right)$

$N=\int D(E) d E \frac{\gamma_{1} f_{1}+\gamma_{2} f_{2}}{\gamma_{1}+\gamma_{2}}$

To estimate the potential energy of the island, SET is represented through the model shown in Fig. 4, where $C_{\mathrm{S}}$, $C_{\mathrm{D}}$, and $C_{\mathrm{G}}$ represent the capacitances established by the 


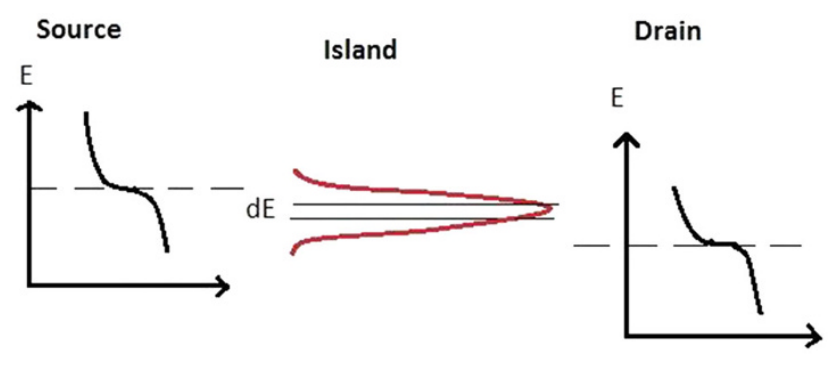

Fig. 3 Broadening of island levels

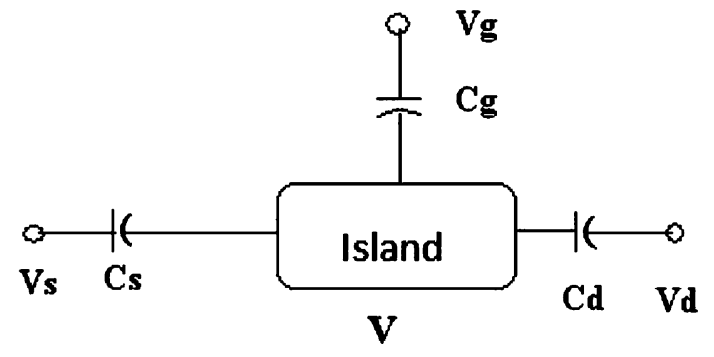

Fig. 4 Capacitance model for SET

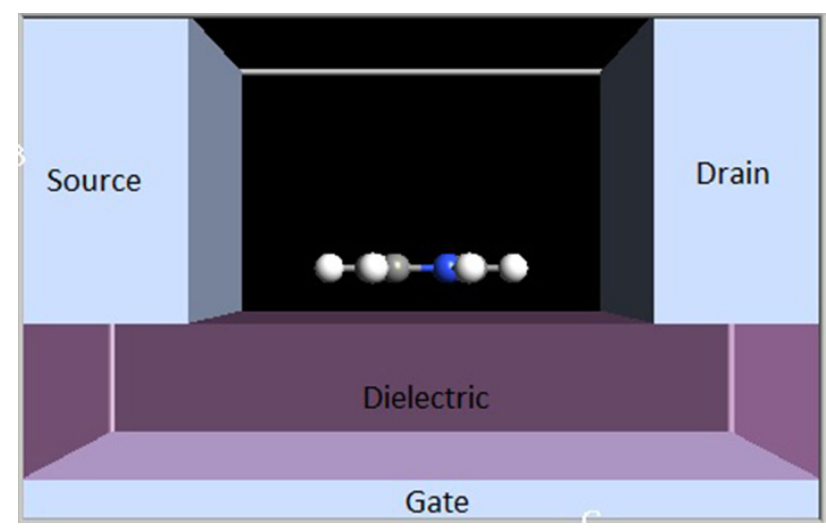

Fig. 5 Impurity added benzene (IAB) based SET source, drain tunnel junctions, and gate dielectric. The amount of charge in the island is given by,

$Q=-q \Delta n=C_{\mathrm{S}} V+C_{\mathrm{G}}\left(V-V_{\mathrm{G}}\right)+C_{\mathrm{D}}\left(V-V_{\mathrm{D}}\right)$

where $q$ is the charge of electron and the potential V (with $\mathrm{V}_{\mathrm{s}}$ grounded) is.

$V=\frac{C_{\mathrm{G}} V_{\mathrm{G}}+C_{\mathrm{D}} V_{\mathrm{D}}}{C_{\mathrm{S}}+C_{\mathrm{G}}+C_{\mathrm{D}}}+\frac{-q \Delta n}{C_{\mathrm{S}}+C_{\mathrm{G}}+C_{\mathrm{D}}}$

Now, the potential energy $U$ of the island can be obtained by multiplying the potential by $-\mathrm{q}$,

$U=-q V=-q\left[\frac{C_{G} V_{G}+C_{D} V_{D}}{C_{S}+C_{G}+C_{D}}+\frac{-q \Delta n}{C_{S}+C_{G}+C_{D}}\right]$

\section{Computational procedure}

In this paper, we have calculated the charging energies of IAB molecules for plotting the charge stability diagrams in both the isolated as well as SET environments, and to analyze the total energy as a function of gate voltage in SET environment. For estimating the charging energies of the molecule, total energies of the molecule for different

Table 1 Calculated charging energies of IAB molecules in isolated and SET environment

\begin{tabular}{llrlrl}
\hline Molecule & Environment & $\mathrm{E}_{\mathrm{I}}^{+1}$ & \multicolumn{1}{l}{$\mathrm{E}_{\mathrm{I}}$} & \multicolumn{1}{c}{$\mathrm{E}_{\mathrm{A}}$} & \multicolumn{1}{l}{$\mathrm{E}_{\mathrm{A}}^{-1}$} \\
\hline $\mathrm{C}_{6} \mathrm{H}_{6}$ & Isolated & -15.72 & -9.13 & -2.32 & -8.38 \\
& SET environment & -9.81 & -7.97 & 1.03 & -0.72 \\
$\mathrm{C}_{5} \mathrm{H}_{6} \mathrm{~B}$ & Isolated & -15.75 & -8.86 & 2.32 & -8.19 \\
& SET environment & -10.11 & -7.07 & 4.52 & -2.2 \\
$\mathrm{C}_{5} \mathrm{H}_{6} \mathrm{~N}$ & Isolated & -15.54 & -4.67 & -1.86 & -8.44 \\
& SET environment & -10.11 & -3.11 & 0.44 & -2.36 \\
$\mathrm{C}_{6} \mathrm{H}_{5} \mathrm{~B}$ & Isolated & -14.93 & -6.87 & 0.001 & -5.98 \\
& SET environment & -10.78 & -5.33 & 2.19 & -0.08 \\
$\mathrm{C}_{6} \mathrm{H}_{5} \mathrm{~N}$ & Isolated & -14.54 & -7.31 & 0.79 & -6.56 \\
& SET environment & -9.13 & -5.61 & 2.96 & -0.74 \\
& & & & &
\end{tabular}

$E_{I}$ ionization potential, $E_{A}$ electron affinity

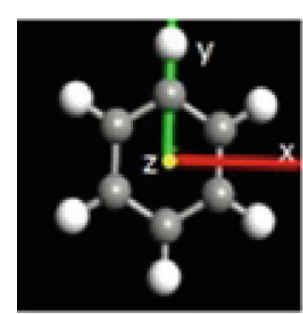

[a] $\mathrm{C}_{6} \mathrm{H}_{6}$

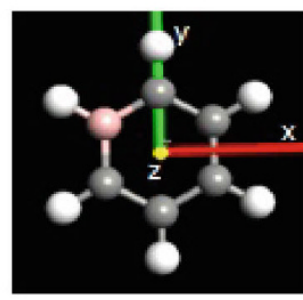

[b] (i) $\mathrm{C}_{5} \mathrm{H}_{6} \mathrm{~B}$

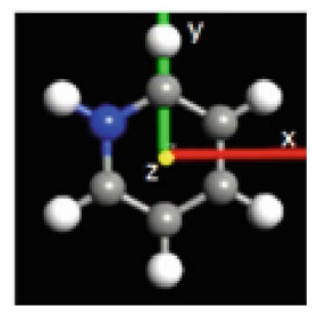

[b] (ii) $\mathrm{C}_{5} \mathrm{H}_{6} \mathrm{~N}$

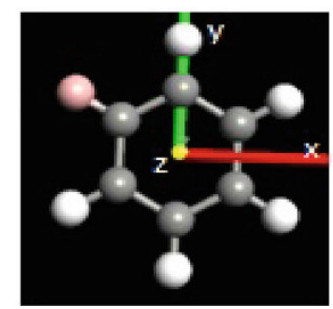

[c] (i) $\mathrm{C}_{6} \mathrm{H}_{5} \mathrm{~B}$

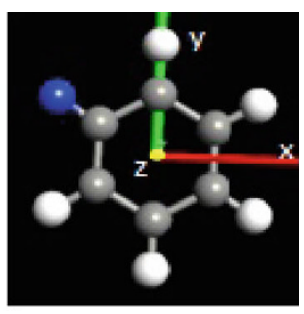

[c] (ii) $\mathrm{C}_{6} \mathrm{H}_{5} \mathrm{~N}$

Fig. 6 Benzene and impurity added benzene (IAB) molecules 


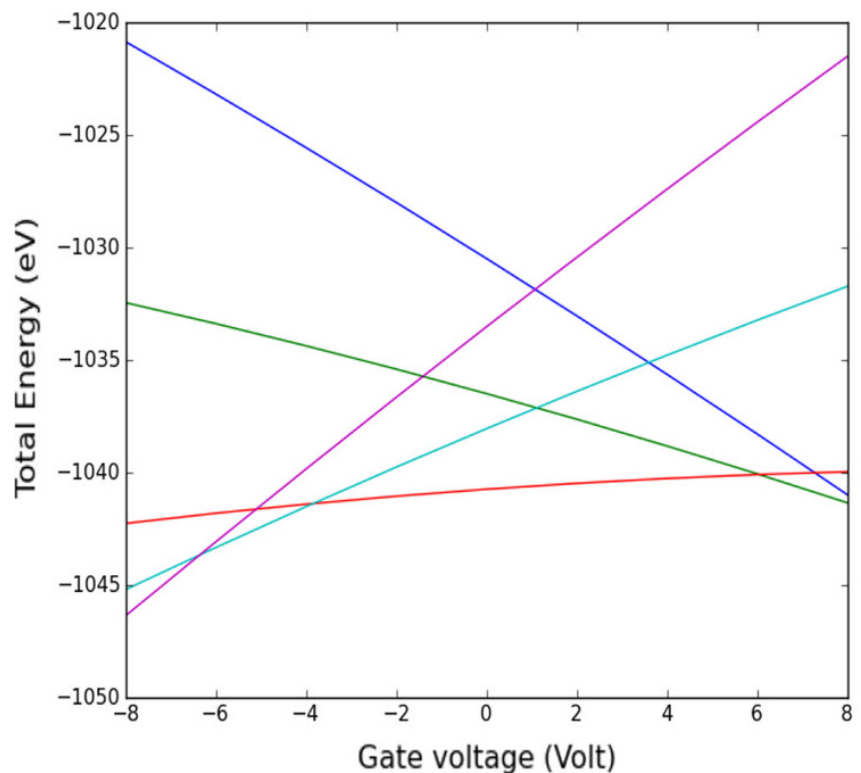

(I) $\mathrm{C}_{6} \mathrm{H}_{6}$

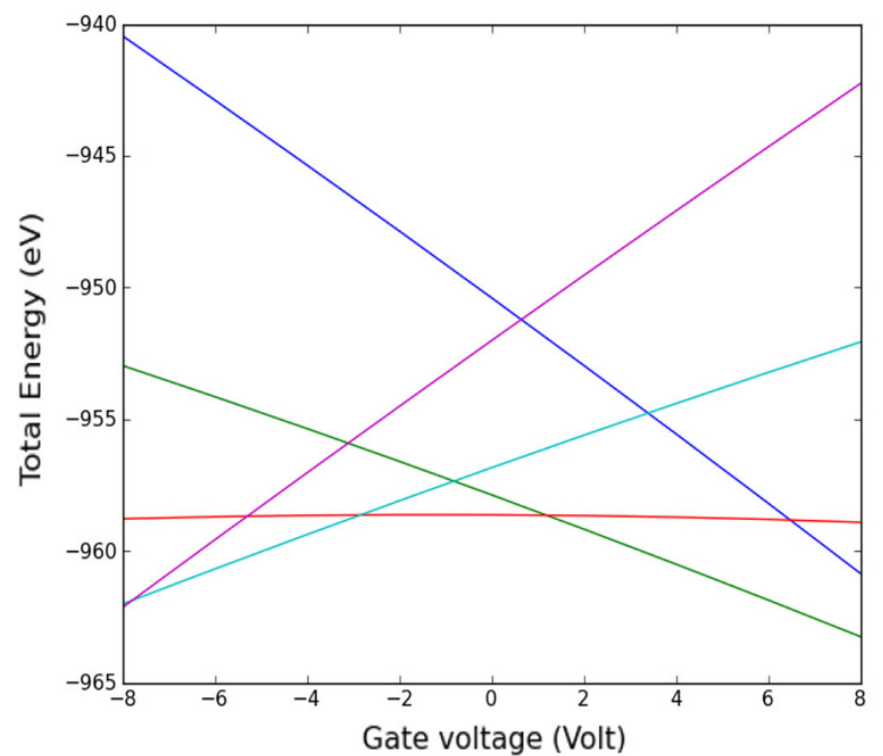

(I) $\mathrm{C}_{5} \mathrm{H}_{6} \mathrm{~B}$

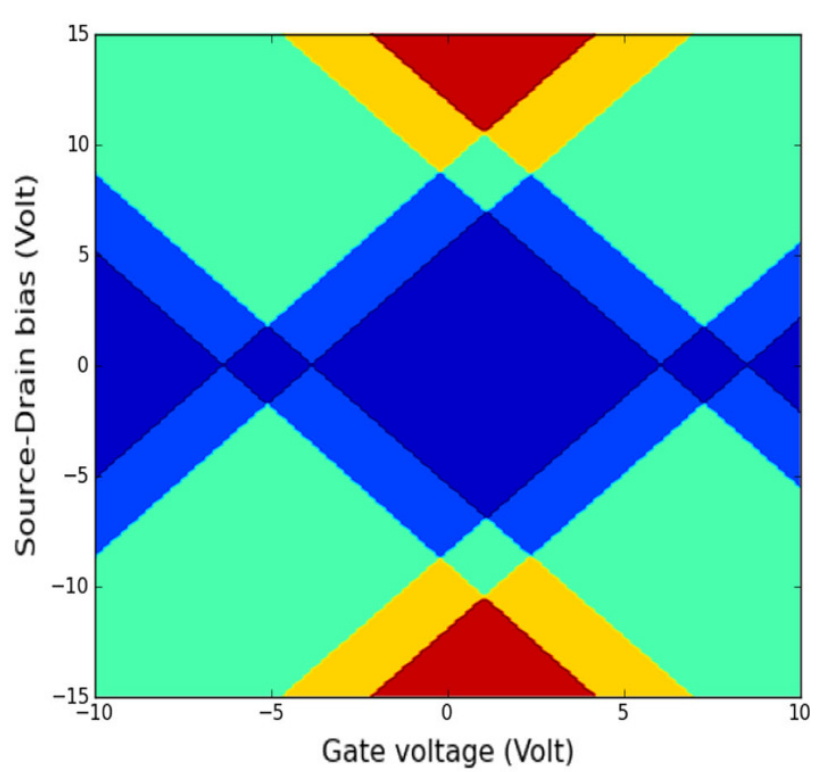

(II) $\mathrm{C}_{6} \mathrm{H}_{6}$

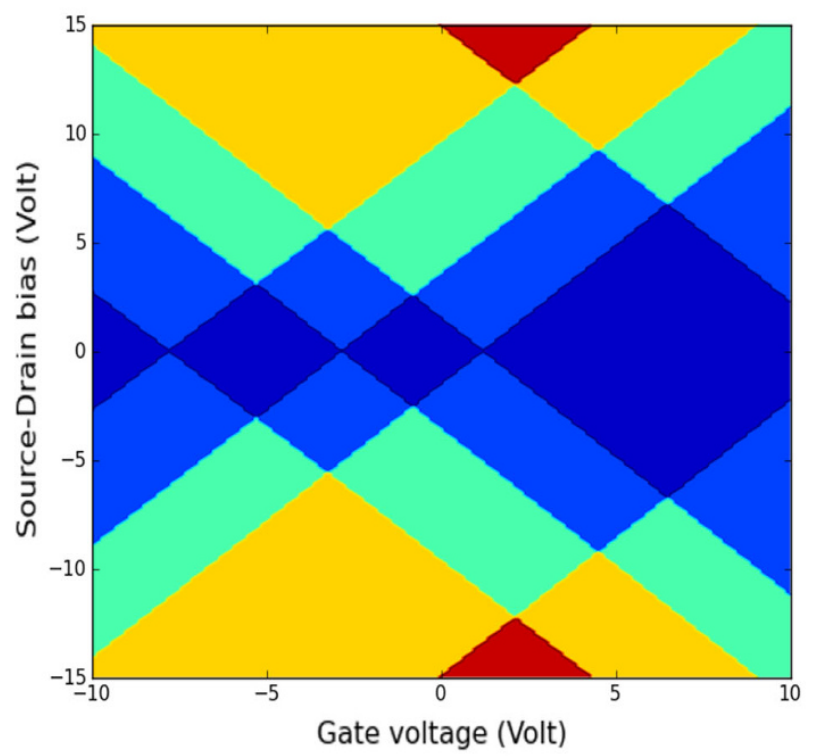

(II) $\mathrm{C}_{5} \mathrm{H}_{6} \mathrm{~B}$

Fig. 7 (I) Total energy as a function of gate potential in SET environment. Each curve represents a charge state: blue [-2], green [-1], red [0], turquoise [1], and violet [2]. (II) Charge stability diagram in SET environment. The colors show the number of Energy levels inside the bias window for a given gate voltage. The colors represent: blue (0), light blue (1), green (2), orange (3), and red (4)

charge states $(-2,-1,0,1,2)$ have been calculated using ATK 11.2.2 Toolkit, which based on density functional theory (DFT) and non-equilibrium Green's function.

Here, in the computation, we have used the IAB molecule as an island in the SET, placed in between the metallic source and drain electrodes above the gate dielectric. The gate dielectric is of $3.8 \AA$ thickness and $10 \varepsilon_{0}$ dielectric constant lies, on a metallic back-gate. The IAB molecule is placed $1.2 \AA$ above the gate dielectric. The separation between IAB to the drain and source electrodes is $2.8 \AA$.
The work function of electrodes is taken as $5.28 \mathrm{eV}$, which is of gold (Riviere 1966). The IAB SET architecture is shown in the Fig. 5. For the analysis of SET, zero volts source-drain bias is applied. To calculate the total energies in the SET environment, we used non-polarized DFT, and local density approximation (LDA) is taken as the exchange correlation function. Basis set double-zeta polarized (DZP) is used for wave functions expansion. Neumen boundary conditions have been applied such that the perpendicular component of the electric field is zero at 


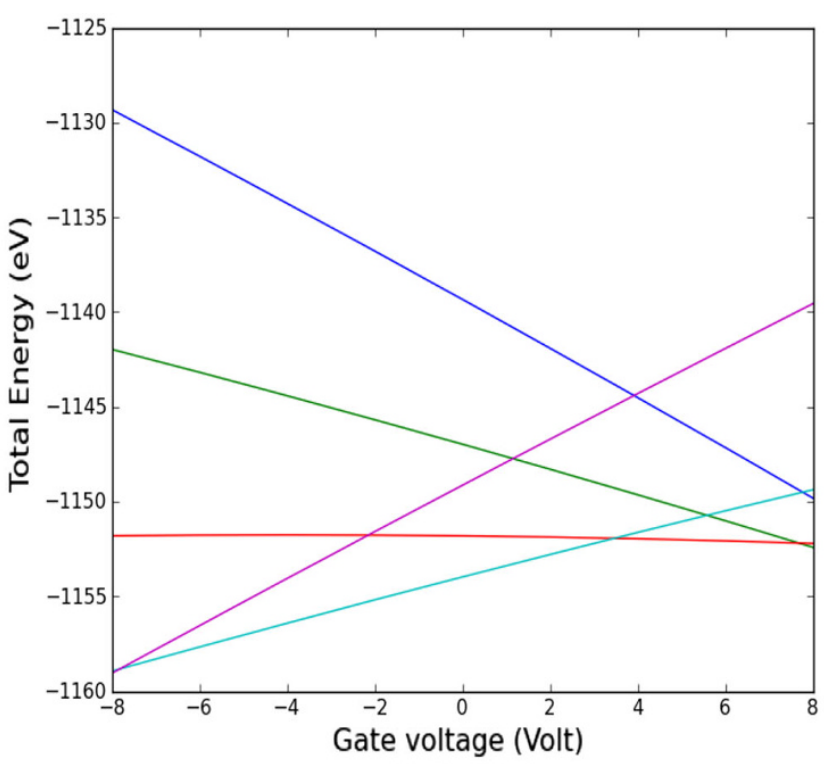

(I) $\mathrm{C}_{5} \mathrm{H}_{6} \mathrm{~N}$

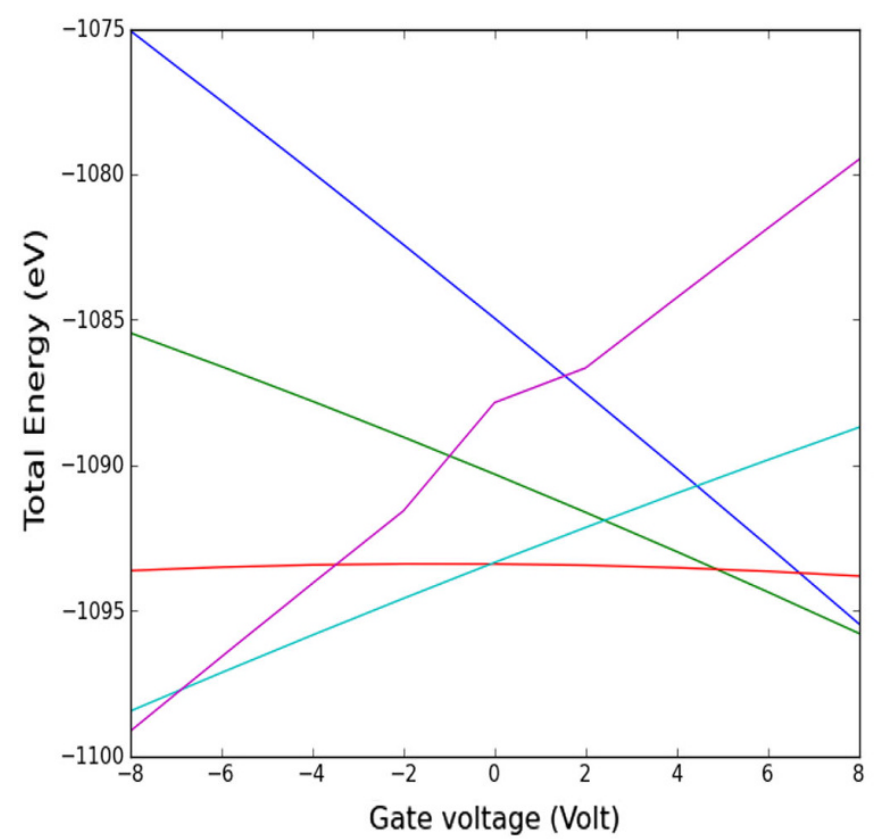

(I) $\mathrm{C}_{6} \mathrm{H}_{5} \mathrm{~B}$

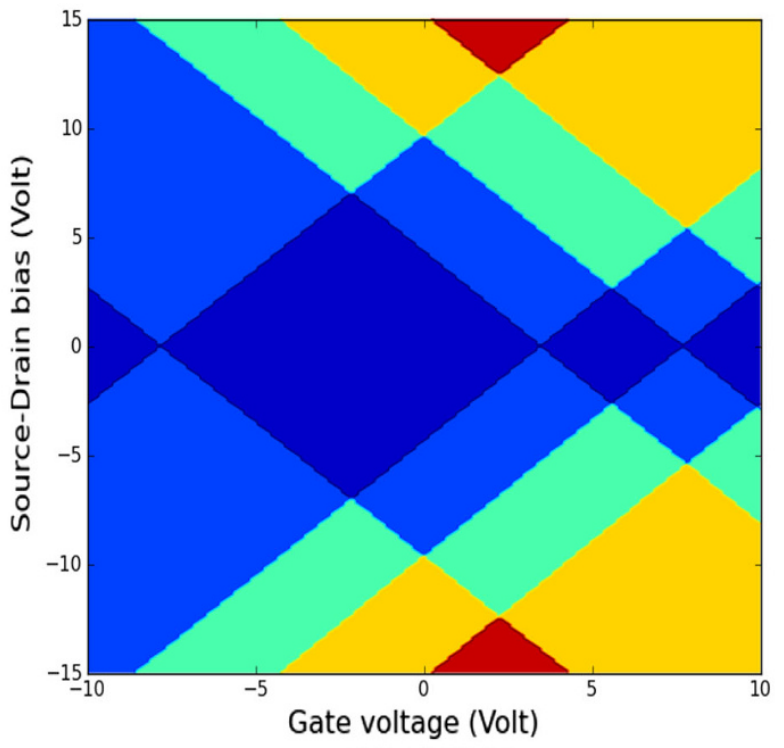

(II) $\mathrm{C}_{5} \mathrm{H}_{6} \mathrm{~N}$

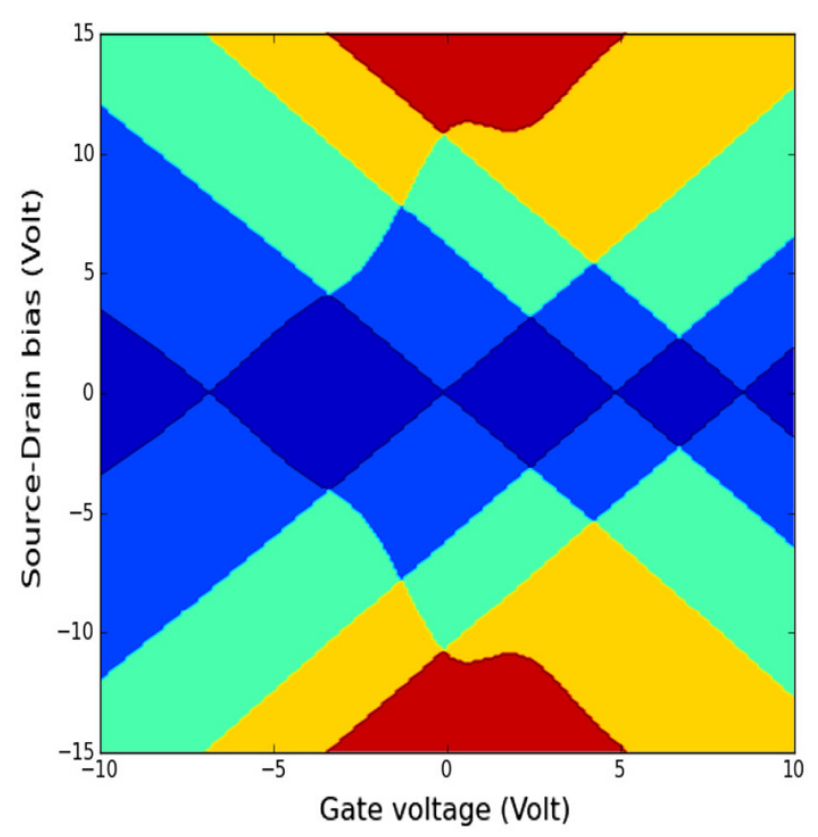

(II) $\mathrm{C}_{6} \mathrm{H}_{5} \mathrm{~B}$

Fig. 7 continued

the boundaries. Further, the energy zero of the molecular energy spectrum is set to absolute energy.

\section{Results and discussion}

We have performed the analysis of benzene by adding boron and nitrogen as impurities in the gas phase (isolated) as well as in SET environment. The impurity addition has been performed in two ways, replacing the last carbon atom of benzene by boron [Fig. 6(b) (i)] and by nitrogen [Fig. 6(b) (ii)] atoms produces $\mathrm{C}_{5} \mathrm{H}_{6} \mathrm{~B}$ (boratabenzene) and $\mathrm{C}_{5} \mathrm{H}_{6} \mathrm{~N}$ (pyridinium), respectively. Replacing the last hydrogen atom of benzene by boron [Fig. 6(c) (i)] and by nitrogen [Fig. 6(c) (ii)] produces $\mathrm{C}_{6} \mathrm{H}_{5} \mathrm{~B}$ and $\mathrm{C}_{6} \mathrm{H}_{5} \mathrm{~N}$, respectively. The charging energies for benzene and $\mathrm{IAB}$ molecules have been computed by calculating the total energies of the Island for different charge states of the molecule $(-2,-1,0,1,2)$ in isolated as well as SET phases and tabulated in Table 1 . The charging energy is defined as 


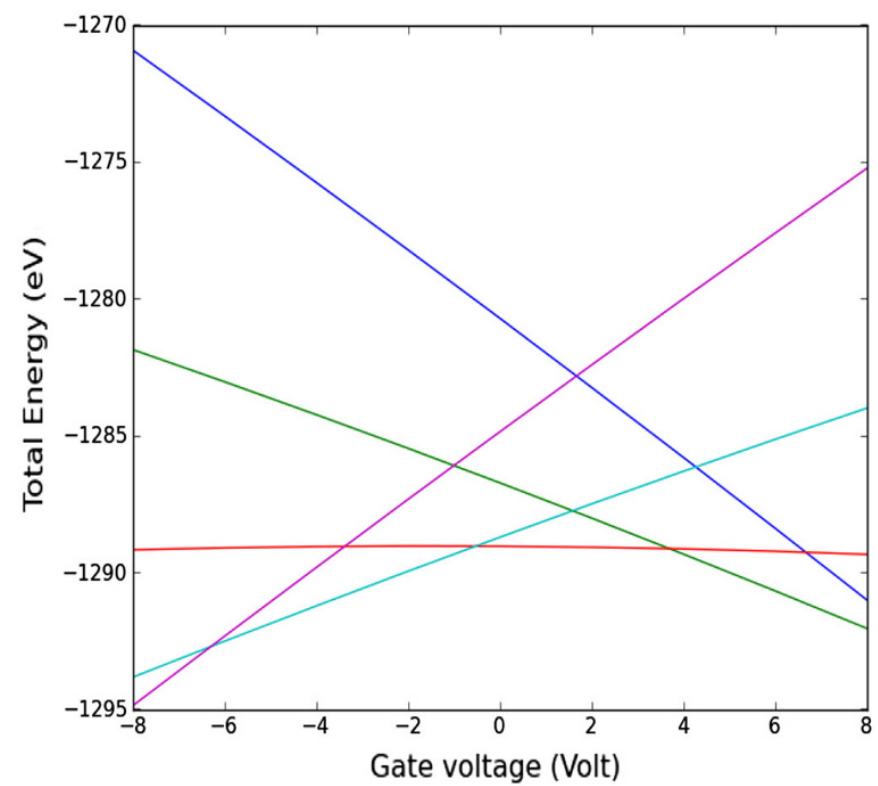

(I) $\mathrm{C}_{6} \mathrm{H}_{5} \mathrm{~N}$

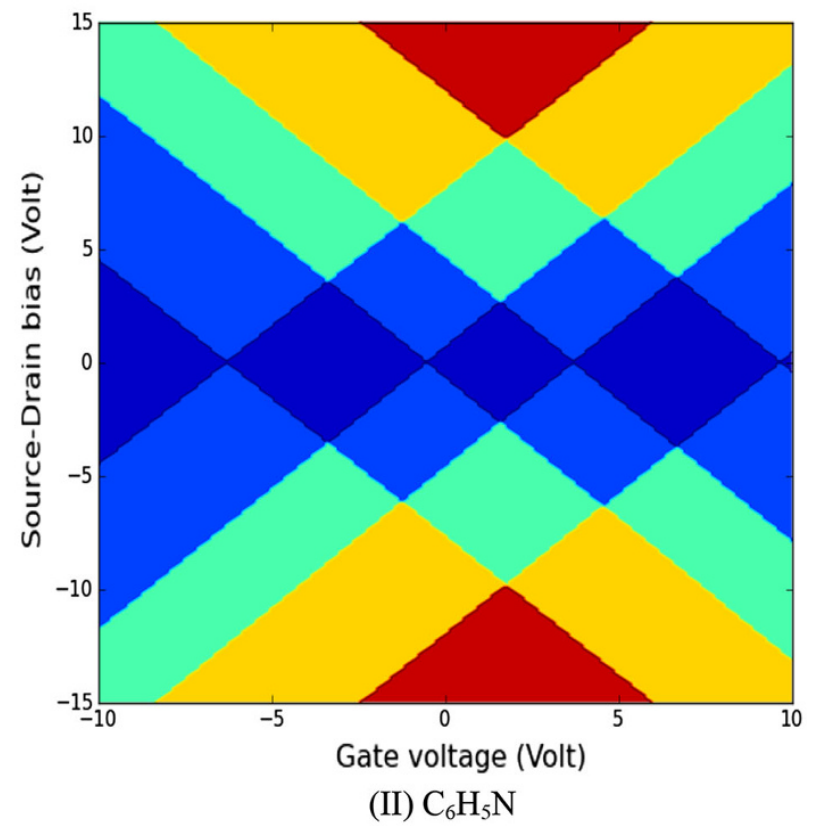

(II) $\mathrm{C}_{6} \mathrm{H}_{5} \mathrm{~N}$

Fig. 7 continued

energy required for charging the tunnel junction with one elementary charge. The ionization potential $\left(E_{\mathrm{I}}\right)$ and electron affinity $\left(E_{\mathrm{A}}\right)$ are calculated using the equations shown below

$E_{I}=E(N)-E(N-1)=E^{0}-E^{+1}$

where $E^{0}$ is the total energy of neutral system having $\mathrm{N}$ electrons, $E^{+1}$ is the total energy of the positive ion having $(\mathrm{N}-1)$ electrons.And,

$E_{A}=E(N)-E(N+1)=E^{0}-E^{-1}$

Similarly, where $E^{-1}$ is the total energy of the negative ion with $(\mathrm{N}+1)$ electrons.

In SET phase, reduction in charging energy is due to the stabilization of the charge on the island by the electrostatic surrounding, and the reason for this stabilization is the use of dielectric material and the IAB molecule lying flat on the dielectric.

In SET, the charging energy of the island can be modified by tuning the gate voltage, which moves the energy levels of the SET in and out of the bias window. This dependence of the SET conductance on the gate voltage and the bias potential can be illustrated with help of charge stability diagram shown as Fig 7-II. These plots consists of color codes, shows the number of energy levels inside the bias window [blue (0), light blue (1), green (2), orange (3), red (4)] for a given value of the source-drain bias and the gate voltage. The conductance of SET is directly related to the number of energy levels inside the bias window. The charge stability diagrams have been plotted for all the IAB
SETs. It can be seen from Fig. 7-I, that in SET environment, at zero gate voltage, $\mathrm{C}_{5} \mathrm{H}_{6} \mathrm{~N}$ has less total energy for its positively charged state (one electron removed from $\mathrm{C}_{5} \mathrm{H}_{6} \mathrm{~N}$ ), thus more stable in its positively charged state compared to other states. $\mathrm{C}_{6} \mathrm{H}_{5} \mathrm{~B}$ is found stable for both the neutral as well as positively charged states at zero gate potential; whereas $\mathrm{C}_{6} \mathrm{H}_{6}, \mathrm{C}_{5} \mathrm{H}_{6} \mathrm{~B}, \mathrm{C}_{6} \mathrm{H}_{5} \mathrm{~N}$ are stable in their neutral states at zero gate potential. Figure 7(II) shows the plotted charge stability diagrams of benzene and all IAB SET. From Fig. 7(II), as expected, benzene and all the IAB SETs are not having any energy level and does not supports conduction at zero gate and source-drain bias. Due to the impurity addition, an improvement in the SET conductance has been observed in terms of gate and source-drain bias. A small change in the gate and source-drain bias does not have much importance for the conduction of benzene based SET, since the energy levels does not come into picture for small change in bias potentials for this SET; whereas, for IAB SETs $\left(\mathrm{C}_{5} \mathrm{H}_{6} \mathrm{~B}, \mathrm{C}_{5} \mathrm{H}_{6} \mathrm{~N}, \mathrm{C}_{6} \mathrm{H}_{5} \mathrm{~B}, \mathrm{C}_{6} \mathrm{H}_{5} \mathrm{~N}\right.$ based SETs $)$, a small change in gate and source-drain potential causes the device to conduct.

\section{Conclusion}

SET with IAB island has been modeled and compared with the pure benzene SET using the ATK 11.2.2 (Atomistix Toolkit, Quantum wise) code. In finding, we observed that at zero gate potential in SET environment, $\mathrm{C}_{5} \mathrm{H}_{6} \mathrm{~N}$ shows more stability in its positively charged state in reference to 
its less total energy in its positively charged state and $\mathrm{C}_{6} \mathrm{H}_{5} \mathrm{~B}$ shows more stability in neutral and positively charged states; whereas $\mathrm{C}_{6} \mathrm{H}_{6}, \mathrm{C}_{5} \mathrm{H}_{6} \mathrm{~B}$, and $\mathrm{C}_{6} \mathrm{H}_{5} \mathrm{~N}$ show more stability in their neutral states only. The charge stability diagrams for the IAB SET have been plotted, which illustrates the dependence of conductance of SET on bias potential and gate voltage. With these findings, we conclude that the work presented here will be of great importance in understanding the role of impurity on overall performance of the SET. The IAB SETs show good improvement in the conductance in comparison to benzene based SET in terms of gate and source-drain bias.

Acknowledgments Authors are thankful to ABV-IIITM, for providing the infrastructural support for carrying out present research work.

Open Access This article is distributed under the terms of the Creative Commons Attribution License which permits any use, distribution, and reproduction in any medium, provided the original author(s) and the source are credited.

\section{References}

Altshuler BL, Lee PA, Webb RA (1991) Mesoscopic phenomena in solids. Elsevier, Amsterdam
Atomistix Toolkit version 11.2.2, Quantum wise A/S (http://quantum wise.com)

Brandbyge M, Mozos JL, Ordejon P, Taylor J, Stokbro K (2002) Density-functional method for nonequilibrium electron transport. Phys Rev B 65:165401

Datta S (2004) "ECE 453 Lecture 39: Coulomb Blockade," http:// nanohub.org/resources/756

Fulton TA, Dolan GJ (1987) Observation of single-electron charging effects in small tunnel junctions. Phys Rev Lett 59:109-112

Grabert H, Devoret MH (1992) Single charge tunneling: coulomb blockade phenomena in nanostructures., NATO Advanced Science Institution Series B, vol. 294Plenum Press, New York

Kumar O, Kaur M (2010) Single electron transistor: applications and problems. Int J VLSI Des Commun Syst (VLSICS) 1(4):24-29

Mollick E (2006) Establishing Moore's law. Annals of the History of Computing, IEEE 28(3):62-75

Neaton JB, Hybertsen MS, Louie SG (2006) Renormalization of molecular electronic levels at metal-molecule interfaces. Phys Rev Lett 97:216405

Parashar S, Srivastava P, Pattanaik M (2012) First-principles study of naphthalene-based single-electron transistor. Appl Nanosci 2(3): $385-388$

Riviere JC (1966) The work function of gold. Appl Phys Lett 8:172

Seldenthuis JS, van der Zant HSJ, Ratner MA, Thijssen JM (2010) Vibrational excitations in weakly coupled single-molecule junctions: a computational analysis. ACS Nano 2:1445-1451

Stokbro K (2010) First-principles modelling of molecular singleelectron transistors. J Phys Chem C 114(48):20461-20465 